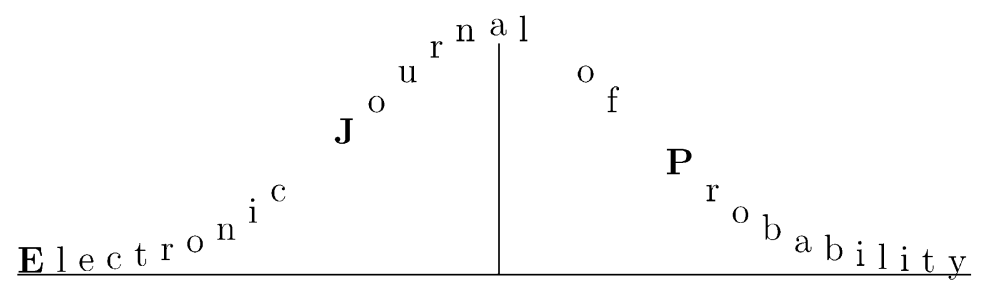

Vol. 6 (2001) Paper no. 23, pages 1-13.

Journal URL

http: //www . math. washington.edu/ ejpecp/

Paper URL

http://www.math. washington.edu/ ${ }^{\sim}$ jpecp/EjpVol6/paper23.abs.html

\title{
RECURRENCE OF DISTRIBUTIONAL LIMITS OF FINITE PLANAR GRAPHS
}

\section{Itai Benjamini}

The Weizmann Institute and Microsoft Research, Mathematics Department

Weizmann Institute of Science, Rehovot 76100, Israel

itai@wisdom.weizmann.ac.il

\section{Oded Schramm}

Microsoft Research, One Microsoft way, Redmond, WA 98052

schramm@microsoft.com

\begin{abstract}
Suppose that $G_{j}$ is a sequence of finite connected planar graphs, and in each $G_{j}$ a special vertex, called the root, is chosen randomly-uniformly. We introduce the notion of a distributional limit $G$ of such graphs. Assume that the vertex degrees of the vertices in $G_{j}$ are bounded, and the bound does not depend on $j$. Then after passing to a subsequence, the limit exists, and is a random rooted graph $G$. We prove that with probability one $G$ is recurrent. The proof involves the Circle Packing Theorem. The motivation for this work comes from the theory of random spherical triangulations.
\end{abstract}

Keywords Random triangulations, random walks, mass trasport, circle packing, volume growth

Mathematics subject classification 2000 82B41, 60J45,05C10

Submitted to EJP on March 14, 2001. Final version accepted on September 19, 2001. 


\section{Introduction}

\section{$1.1 \quad$ Random triangulations}

In recent years, physicists were interested in the study of random surfaces [ADJ97]. Random triangulations turned out to be a useful model for exact calculations, non rigorous arguments, and Monte-Carlo simulations regarding the geometry of random surfaces and the behaviour of physical systems on these surfaces. From a mathematical viewpoint, natural measures that were considered are the uniform measures on isomorphism classes of triangulations of the sphere with a fixed number of vertices. In $\left[\mathrm{AAJ}^{+} 98, \mathrm{ANR}^{+} 98\right.$ ] diffusion on some random surfaces and random walks on random triangulations including the uniform measure have been considered. It was suggested there that the probability for the random walk to be at time $t$ at its starting vertex should decay like $t^{-1}$, provided that $t$ is not too large relative to the size of the triangulation, and that the mean square displacement at time $t$ is $t^{1 / 2}$. Motivated by these observations, we decided to study the recurrence versus transience dichotomy for limits of random rooted spherical triangulations. It turned out that in the end the results apply to the more general setting of planar bounded-degree graphs. It will be proven that under the assumption of a uniform bound on the vertex degrees, limits of finite planar graphs are recurrent (provided that the root is chosen uniformly).

\subsection{Limits of graphs}

In addition to studying asymptotic properties of large random objects, it is mathematically appealing and natural to introduce a limiting infinite object and study its properties. In order to define the limit of a sequence of (possibly random) triangulations or graphs, it is necessary to keep track of a basepoint; or a root.

A rooted graph is just a pair $(G, o)$, where $G$ is a (connected) graph and $o$ is a vertex in $G$. A rooted graph $(G, o)$ is isomorphic to $\left(G^{\prime}, o^{\prime}\right)$ if there is an isomorphism of $G$ onto $G^{\prime}$ which takes $o$ to $o^{\prime}$. In this paper, we only consider locally finite graphs; that is, each vertex has finitely many neighbors.

The space $\mathcal{X}$ of isomorphism classes of rooted connected (locally finite) graphs has a natural topology, which is induced by the following metric. Let $(G, o),\left(G^{\prime}, o^{\prime}\right) \in \mathcal{X}$. For $r=1,2, \ldots$, let $B_{G}(o, r)$ be the closed ball of radius $r$ about $o$ in $G$, and similarly for $G^{\prime}$. Let $k$ be the supremum of all $r$ such that $\left(B_{G}(o, r), o\right)$ and $\left(B_{G^{\prime}}\left(o^{\prime}, r\right), o^{\prime}\right)$ are isomorphic as rooted graphs, and set $d\left((G, o),\left(G^{\prime}, o^{\prime}\right)\right):=2^{-k}$, where $2^{-\infty}:=0$. Then $d$ is a metric on $\mathcal{X}$. Moreover, it is easy to verify that for every $M<\infty$ the subspace $\mathcal{X}_{M} \subset \mathcal{X}$ of graphs with degrees bounded by $M$ is compact in this topology.

Suppose that $H$ is a finite connected graph. We cannot think of $H$ as an element of $\mathcal{X}$, unless a root $o$ is chosen. The most natural way to choose a root is to make the choice random, and uniform among the vertices of $H$. In this way, a finite unrooted graph $H$ corresponds to a probability measure $\mu_{H}$ on $\mathcal{X}$. More explicitly, for every Borel subset 
$\mathcal{A} \subset \mathcal{X}, \mu_{H}(\mathcal{A})$ is equal to the probability that $(H, o) \in \mathcal{A}$ when $o$ is chosen uniformly and randomly among the vertices of $H$.

Suppose that $(G, o)$ is a random rooted finite graph. (This means that $(G, o)$ is a sample from a Borel probability measure $\mu$ on $\mathcal{X}$, which is called the law of $(G, o)$, and $\mu$ is supported on the set of finite graphs.) Then $(G, o)$ is unbiased if its law is in the closed convex hull of the measures $\mu_{H}$. In other words, for every finite graph $H$, conditioned on the event that $G$ is isomorphic to $H$, the distribution of $(G, o)$ is $\mu_{H}$ (provided that $G$ is isomorphic to $H$ with positive probability). Informally, a random rooted finite graph $(G, o)$ is unbiased, if given $G$ the root $o$ is uniformly distributed among the vertices $V(G)$. Let $(G, o)$ and $\left(G_{1}, o_{1}\right),\left(G_{2}, o_{2}\right), \ldots$ be random connected rooted graphs. We say that $(G, o)$ is the distributional limit of $\left(G_{j}, o_{j}\right)$ as $j \rightarrow \infty$ if for every $r>0$ and for every finite rooted graph $\left(H, o^{\prime}\right)$, the probability that $\left(H, o^{\prime}\right)$ is isomorphic to $\left(B_{G_{j}}\left(o_{j}, r\right), o_{j}\right)$ converges to the probability that $\left(H, o^{\prime}\right)$ is isomorphic to $\left(B_{G}(o, r), o\right)$. This is equivalent to saying that the law of $(G, o)$, which is a probability measure on $\mathcal{X}$, is the weak limit of the law of $\left(G_{j}, o_{j}\right)$ as $j \rightarrow \infty$.

It is easy to see, by compactness or diagonalization, that if $\left(G_{j}, o_{j}\right) \in \mathcal{X}_{M}$ a.s., $M<\infty$, then there is always a subsequence of the sequence $\left(G_{j}, o_{j}\right)$ having a distributional limit.

Theorem 1.1. Let $M<\infty$, and let $(G, o)$ be a distributional limit of rooted random unbiased finite planar graphs $G_{j}$ with degrees bounded by $M$. Then with probability one $G$ is recurrent.

Remarks. To illustrate the theorem, the reader may wish to consider the case where each $G_{j}$ is a finite binary tree of depth $j$. (In that case the distance of the root from the leaves of the tree is approximately a geometric random variable. Hence, $G$ is not the 3-regular tree.)

The assumption of planarity in the theorem is necessary, as can be seen by considering the intersection of $\mathbb{Z}^{3}$ with larger and larger balls. In this case, since the surface area to volume ratio tends to zero, the root is not likely to be close to the boundary. The distributional limit will then be $\left(\mathbb{Z}^{3}, 0\right)$ a.s. Also, the 3 -regular tree can be obtained as the a.s. limit of unbiased finite graphs (3 regular graphs with girth going to infinity).

A natural extension of the collection of all planar graphs is the collection of all graphs with an excluded minor. It is reasonable to guess that if $H$ is any graph and one replaces the assumption of planarity by the assumption that all $G_{j}$ do not have $H$ as a minor, then the theorem still holds, because many theorems on planar graphs generalize to excluded minor graphs (see, e.g., [Tho99]).

Because of the assumption of a uniform bound on the degrees, the interesting case when $G_{n}$ is uniformly distributed among all isomorphism classes of triangulations of size $n$ is unfortunately not included. We conjecture that the theorem still holds for limits of these measures. (See [Ma199, GW00] for a study of the degree distribution for those measures.) If $G_{n}$ is uniformly distributed among spherical triangulations of size $n$ with degree at most $M$, then the theorem above does apply to any (subsequential) limit of $G_{n}$. 
A key to almost all the rigorous results regarding random surfaces and triangulations is the enumeration techniques, which originated with the fundamental work of Tutte [Tut62]. Schaeffer [Sch99] found a simpler proof for Tutte's enumeration, and thereby produced a good sampling algorithm. In contrast, our approach in this paper makes no use of enumeration. The proof of 1.1 is based on the theory of circle packings with specified combinatorics.

Following is another statement of the theorem from a slightly different perspective. Let $G$ be a finite graph and let $X_{0}, X_{1}, \ldots$ be simple random walk on $G$ started from a random-uniform vertex $X_{0} \in V(G)$. Let $\phi(n, G)$ be the probability that $X_{j} \neq X_{0}$ for all $j=1,2, \ldots, n$, and set

$$
\phi_{M}(n):=\sup \left\{\phi(n, G): G \in \mathcal{G}_{M}\right\},
$$

where $\mathcal{G}_{M}$ denotes the collection of finite planar graphs with maximum degree at most $M$.

Corollary 1.2. For all $M<\infty$,

$$
\lim _{n \rightarrow \infty} \phi_{M}(n)=0
$$

Proof. Let $h:=\inf _{n \in \mathbb{N}} \phi_{M}(n)$. Then there is a sequence of graphs $G_{j} \in \mathcal{G}_{M}$ with $\phi\left(n, G_{j}\right) \geq \phi\left(j, G_{j}\right) \geq h / 2$ for all $n \leq j$. Let $(G, o)$ be a distributional limit of a subsequence of $\left(G_{j}, o_{j}\right)$, where $o_{j}$ is unbiased in $V\left(G_{j}\right)$. Then for every $n \in \mathbb{N}$ the probability that simple random walk on $G$ started from $o$ will not revisit $o$ in the first $n$ steps is at least $h / 2$. By the theorem, $G$ is recurrent, and hence $h=0$, as required.

Problem 1.3. Determine the rate of decay of $\phi_{M}(n)$ as a function of $n$.

The example of an $n \times n$ square in the grid $\mathbb{Z}^{2}$ shows that $\inf _{n} \phi_{M}(n) \log n>0$. It might be reasonable to guess that $\phi_{M}(n)$ decays like $c(M) / \log n$.

In the next section, a proof of Theorem 1.1 is given, and the last section is devoted to miscellaneous remarks, including examples of planar triangulations with uniform growth $r^{\alpha}, \alpha \notin\{1,2\}$.

\section{Recurrence}

Theorem 1.1 will be proved using the theory of combinatorially specified circle packings. At the foundation of this theory is the Circle-Packing Theorem, which states that for every finite planar graph $G$ there is a disk-packing $P$ in the plane whose tangency graph is $G$; which means that the disks in $P$ are indexed by the vertices $V(G)$ of $G$ and two disks are tangent iff the corresponding vertices share an edge. When $G$ is the 1-skeleton of a triangulation of the sphere, the packing $P$ is unique up to Möbius transformations. 
The Circle Packing Theorem was first proved by Koebe [Koe36] and later various generalizations have been obtained. See, e.g., [dV91] for a proof of this result. The Circle Packing Theorem has been instrumental in answering some problems about the geometry and potential theory of planar graphs [MP94, MT90, BS96, JS00]. (The relations between circle-packings and analytic function theory are important, fascinating, and much studied, but are not as relevant to the present paper.) Some surveys of the theory of combinatorially specified circle packings are also available [Ber92, Sac94, Ste99].

The major step in the proof of Theorem 1.1 is the case of triangulations, that is,

Proposition 2.1 (The triangulation case). Let $M<\infty$, and let $(T, o)$ be a distributional limit of rooted random unbiased (finite) triangulations of the sphere $T_{j}$ with degrees bounded by $M$. Then with probability one $T$ is recurrent.

Proof. We assume, as we may, that $T$ is a.s. infinite. By the Circle-Packing Theorem, for each $j$, there is a disk-packing $P^{j}$ in the plane with tangency graph $T_{j}$. Since $T^{j}$ is random, also $P^{j}$ is random. (Actually, the randomness in $T^{j}$ plays no role in the proof, and we could assume that $T^{j}$ is deterministic. The essential randomness is that of $o_{j}$.) We choose $P^{j}$ to be independent of $o_{j}$ given $T^{j}$. Our immediate goal is to take an appropriate limit of the disk-packings $P^{j}$ to obtain a disk-packing $P$ with tangency graph $T$.

There is a unique triangle $t_{j}$ in $T_{j}$ whose vertices correspond to three disks of $P^{j}$ which intersect the boundary of the unbounded component of $\mathbb{R}^{2} \backslash P^{j}$. For every vertex $v \in$ $V\left(T_{j}\right) \backslash t_{j}$, the disks corresponding to neighbors of $v$ in $T_{j}$ surround the disk $P_{v}^{j}$ like petals of a flower, as in Figure 2.1. By shrinking the packing, if necessary, assume that $P^{j}$ is contained in the unit disk $B(0,1)$.

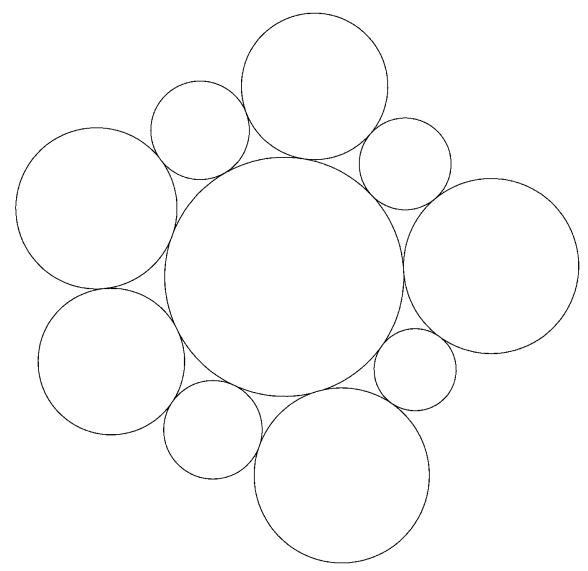

Figure 2.1: The Ring Lemma setup.

Let $\hat{P}^{j}$ be the image of the packing $P^{j}$ under the map $z \mapsto a z+b$, where $a \in(0, \infty)$ and $b \in \mathbb{R}^{2}$ are chosen so that $\hat{P}_{o_{j}}^{j}$ is the unit disk $B(0,1)$. 
Here is a simple but important fact about disk packings, known as the Ring Lemma [RS87]. If a disk $P_{0}$ is surrounded by $n$ other disks $P_{1}, \ldots, P_{n}$, as in Figure 2.1, then the ratio $r_{0} / r_{1}$ between the radius of $P_{0}$ and the radius of $P_{1}$ is bounded from above by a constant which depends only on $n$. Since the vertex degrees in the triangulations $T_{j}$ are all bounded by $M$, it follows that for every $d$ there is some upper bound $c=c(d, M)$ for the ratio $r / r^{\prime}$ between any two radii of disks corresponding to vertices at combinatorial distance at most $d$ from $o_{j}$, provided that $o_{j}$ is at combinatorial distance at least $d+1$ from $t_{j}$. Because $\left|V\left(T_{j}\right)\right| \rightarrow \infty$ as $j \rightarrow \infty$, with probability tending to $1, P_{o_{j}}^{j}$ is not one of the boundary disks corresponding to vertices of $t_{j}$. Moreover, by the uniform bound on the degrees, the combinatorial distance in $T^{j}$ from $o_{j}$ to $t_{j}$ tends to infinity in probability as $j \rightarrow \infty$, because for every $r \geq 1$ the number of vertices of $T^{j}$ at distance at most $r$ from $t_{j}$ is bounded, and hence $o_{j}$ is unlikely to be there. We may therefore conclude that for every $d$ there is a constant $c=c(d)$ so that with probability tending to 1 all the disks in $\hat{P}^{j}$ corresponding to vertices at combinatorial distance at most $d$ from $o_{j}$ have radii in $[1 / c, c]$. By compactness, we may take a subsequence of $j$ tending to $\infty$ so that the law of $\hat{P}^{j}$ tends (in the appropriate topology) to a random disk packing $P$ whose tangency graph is $T$. Assume, with no loss of generality, that there is no need to pass to a subsequence.

An accumulation point of $P$ is a point $z \in \mathbb{R}^{2}$ such that every open set containing $z$ intersects infinitely many disks in $P$. Below, we prove the following result.

Proposition 2.2. With probability 1 , there is at most one accumulation point in $\mathbb{R}^{2}$ of the packing $P$.

Proof of Proposition 2.1, continued. Assuming 2.2, the proof is completed as follows. In [HS95, Thms. 2.6, 3.1.(1), 8.1] and independently in [McC98] it was shown that if $G$ is a bounded degree tangency graph of a disk-packing in the plane which has no accumulation points in the plane, then $G$ is recurrent. This completes the proof if $P$ has no accumulation points in $\mathbb{R}^{2}$. If $P$ has one accumulation point $p \in \mathbb{R}^{2}$, then consider the subgraph $G_{1}$ of $T$ spanned by the vertices corresponding to disks contained in the disk $B(p, 1)$ of radius 1 about $p$. By inverting in the circle of radius 1 about $p$, it follows that $G_{1}$ is recurrent. (Note that the above quoted results do not require the graph to be the 1-skeleton of a triangulation.) Similarly, the subgraph $G_{2}$ spanned by the vertices of $T$ corresponding to disks that intersect the complement of $B(p, 1)$ is also recurrent. Since $V(T)=V\left(G_{1}\right) \cup V\left(G_{2}\right)$ and the boundary separating $G_{1}$ and $G_{2}$ is finite, it follows that $T$ is recurrent.

For the proof of Proposition 2.2, a lemma will be needed, but some notations must be introduced first. Suppose that $C \subset \mathbb{R}^{2}$ is a finite set of points. (In the application below, $C$ will be the set of centers of disks in $P^{j}$.) When $w \in C$, we define its isolation radius as $\rho_{w}:=\inf \{|v-w|: v \in C \backslash\{w\}\}$. Given $\delta \in(0,1), s>0$ and $w \in C$, we say that $w$ is $(\delta, s)$-supported if in the disk of radius $\delta^{-1} \rho_{w}$, there are more than $s$ points of $C$ outside of every disk of radius $\delta \rho_{w}$; that is, if

$$
\inf _{p \in \mathbb{R}^{2}}\left|C \cap B\left(w, \delta^{-1} \rho_{w}\right) \backslash B\left(p, \delta \rho_{w}\right)\right| \geq s
$$


Lemma 2.3. For every $\delta \in(0,1)$ there is a constant $c=c(\delta)$ such that for every finite $C \subset \mathbb{R}^{2}$ and every $s \geq 2$ the set of $(\delta, s)$-supported points in $C$ has cardinality at most $c|C| / s$.

Proof. Let $k \in\{3,4,5, \ldots\}$. Consider a bi-infinite sequence $\mathfrak{S}=\left(\mathfrak{S}_{n}: n \in \mathbb{Z}\right)$ of square-tilings $\mathfrak{S}_{n}$ of the plane, where for all $n \in \mathbb{Z}$ all the squares in the tiling $\mathfrak{S}_{n+1}$ have the same size and each square in the tiling $\mathfrak{S}_{n+1}$ is tiled by $k^{2}$ squares in the tiling $\mathfrak{S}_{n}$. Let $\hat{\mathfrak{S}}$ denote the collection of all the squares in all the tilings $\mathfrak{S}_{n}$. Assume that no point of $C$ lies on the boundary of a square in $\hat{\mathfrak{S}}$. For every $n \in \mathbb{Z}$, say that a square $S \in \mathfrak{S}_{n}$ is $s$-supported if for every square $S^{\prime} \in \mathfrak{S}_{n-1}$, we have $\left|C \cap S \backslash S^{\prime}\right| \geq s$. To estimate the number of $s$-supported squares in $\hat{\mathfrak{S}}$, we now define a "flow" $f$ on $\hat{\mathfrak{S}}$. Set

$$
f\left(S^{\prime}, S\right):= \begin{cases}\min \left\{s / 2,\left|S^{\prime} \cap C\right|\right\} & S \in \mathfrak{S}_{n+1}, S^{\prime} \in \mathfrak{S}_{n}, S^{\prime} \subset S, \\ 0 & S \in \mathfrak{S}_{n+1}, S^{\prime} \in \mathfrak{S}_{n}, S^{\prime} \not \subset S, \\ -f\left(S, S^{\prime}\right) & S \in \mathfrak{S}_{n}, S^{\prime} \in \mathfrak{S}_{n+1}, \\ 0 & S \in \mathfrak{S}_{n}, S^{\prime} \in \mathfrak{S}_{m},|m-n| \neq 1 .\end{cases}
$$

Let $a \in \mathbb{Z}$ be small enough so that each square of $\mathfrak{S}_{a}$ contains at most one point of $C$, and let $b \in \mathbb{Z}, b>a$. Then

$$
\sum_{S^{\prime} \in \mathfrak{S}_{a}} \sum_{S \in \mathfrak{G}_{a+1}} f\left(S^{\prime}, S\right)=|C|, \quad \sum_{S^{\prime} \in \mathfrak{G}_{b}} \sum_{S \in \mathfrak{G}_{b+1}} f\left(S^{\prime}, S\right) \geq 0 .
$$

Therefore

$$
\sum_{n=a+1}^{b} \sum_{S \in \mathfrak{S}_{n}} \sum_{S^{\prime} \in \hat{\mathfrak{S}}} f\left(S^{\prime}, S\right) \leq|C|,
$$

because for every pair $S^{\prime} \in \mathfrak{S}_{n}, S \in \mathfrak{S}_{n+1}$ with $n \in\{a+1, \ldots, b-1\}$, the corresponding two terms $f\left(S, S^{\prime}\right)$ and $f\left(S^{\prime}, S\right)$ both appear on the left hand side and they cancel each other. By the definition of $f$, for every $S \in \hat{\mathfrak{S}}$ we have $\sum_{S^{\prime} \in \hat{\mathfrak{S}}} f\left(S^{\prime}, S\right) \geq 0$. On the other hand, if $S \in \hat{\mathfrak{S}}$ is $s$-supported, then $\sum_{S^{\prime} \in \hat{\mathfrak{S}}} f\left(S^{\prime}, S\right) \geq s / 2$. Therefore, (2.1) implies that the number of $s$-supported squares in $\hat{\mathfrak{S}}$ is at most $2|C| / s$.

To estimate the number of $(\delta, s)$-supported points of $C$, we will compare it with the expected number of $s$-supported squares in $\widehat{\mathfrak{S}}$, where $\mathfrak{S}$ is chosen randomly, as follows. Take $k:=\left\lceil 20 \delta^{-2}\right\rceil$ as the parameter for the sequence $\mathfrak{S}$. Let $\mathfrak{S}$ have the distribution such that the distribution of $\hat{\mathfrak{S}}$ is invariant under translation and rescaling, and such that the diameter of any square in $\mathfrak{S}_{0}$ is in the range $[1, k)$.

To be explicit, we now construct this distribution. Let $\left(\alpha_{n}, n \in \mathbb{Z}\right)$ be a sequence of independent random variables with each $\alpha_{n}$ uniform in $\{0,1, \ldots, k-1\}^{2}$. Let $\beta$ be uniform in $[0, \log k)$ and independent from the sequence $\left(\alpha_{n}\right)$. Then we may take

$$
\mathfrak{S}_{n}=\left\{e^{\beta} k^{n}\left([j, j+1] \times\left[j^{\prime}, j^{\prime}+1\right]\right)+e^{\beta} \sum_{m=-\infty}^{n-1} k^{m} \alpha_{m}: j, j^{\prime} \in \mathbb{Z}\right\} .
$$


Let $N$ be the number of $(\delta, s)$-supported points in $C$. Say that a point $w \in C$ is a city in a square $S \in \hat{\mathfrak{S}}$ if the edge-length of $S$ is in the range $\left[4 \delta^{-1} \rho_{w}, 5 \delta^{-1} \rho_{w}\right]$ and the distance from $w$ to the center of $S$ is at most $\delta^{-1} \rho_{w}$. It is easy to see that there is a constant $c_{0}=c_{0}(k)>0$, which does not depend on $C$ or $w$, such that $w$ is a city for some square of $\hat{\mathfrak{S}}$ with probability at least $c_{0}$. By the above choice of $k$, if $w$ is a city in $S$ and $w$ is $(\delta, s)$-supported, then $S$ is $s$-supported. Consequently, the expected number of pairs $(w, S)$ such that $w$ is a city in $S$ and $S$ is $s$-supported is at least $c_{0} N$. However, by area considerations it is clear that there is a constant $c_{1}=c_{1}(\delta)$ such that any square $S$ cannot have more than $c_{1}$ cities in it. Hence, the expected number of $s$-supported squares is at least $c_{0} N / c_{1}$. However, we have seen above that the number of $s$-supported squares is at most $2|C| / s$. Hence $N \leq 2 c_{0}{ }^{-1} c_{1}|C| / s$, which proves the lemma.

Proof of Proposition 2.2. Suppose that there is a positive probability that $P$ has two distinct accumulation points in $\mathbb{R}^{2}$. Then there is a $\delta \in(0,1)$ and an $\epsilon>0$ such that with probability at least $\epsilon$ there are two accumulation points $p_{1}, p_{2}$ in $B\left(0, \delta^{-1}\right)$ such that $\left|p_{1}-p_{2}\right| \geq 3 \delta$. But this implies that for arbitrarily large $s$ and for infinitely many $j$ there is probability at least $\epsilon$ that the center of $P_{o_{j}}^{j}$ is $(\delta, s)$-supported in the set $C^{j}$ of centers of the disks in $P^{j}$, contradicting Lemma 2.3 .

Proof of Theorem 1.1. We claim that there is a constant $c$ such that for all $j=1,2, \ldots$ there is a triangulation $T_{j}$ of the sphere with maximum vertex degree at most $c M$, which contains a subgraph isomorphic to $G_{j}$, and such that $\left|V\left(T_{j}\right)\right| \leq c\left|V\left(G_{j}\right)\right|$. Indeed, embed $G_{j}$ in the plane, and let $f$ be a face of this embedding. Let $v_{0}, \ldots, v_{k-1}$ be the vertices on the boundary of $f$, in clockwise order. First, suppose that $v_{j} \neq v_{i}$ for $j \neq i$, and that $v_{j}$ does not neighbor with $v_{i}$ unless $|i-j|=1$ or $\{i, j\}=\{0, k-1\}$. In that case, we triangulate $f$ by a zigzag pattern; that is, put into $T_{j}$ the edges $\left[v_{j}, v_{k-j}\right], j=$ $1,2, \ldots,\lfloor k / 2\rfloor-1$ and the edges $\left[v_{j}, v_{k-1-j}\right], j=1,2, \ldots,\lfloor(k-1) / 2\rfloor-1$. Otherwise, first put inside $f$ a cycle of length $k, u_{0}, \ldots, u_{k-1}$, then put the edges $\left[u_{j}, v_{j}\right], j=0, \ldots, k-1$ and $\left[u_{j}, v_{j+1}\right], j=0, \ldots, k-2$ and $\left[u_{k-1}, v_{0}\right]$, and then triangulate the face bounded by the new cycle. It is easy to verify that when this construction is applied to every face $f$ of the embedding of $G$, the resulting triangulation satisfies the required conditions for some appropriate constant $c$.

Let $o_{j}$ be a vertex chosen uniformly in $V\left(T_{j}\right)$. Since, $\left|V\left(T_{j}\right)\right| \leq c\left|V\left(G_{j}\right)\right|$, we have $\mathbf{P}\left[o_{j} \in V\left(G_{j}\right)\right] \geq 1 / c$. Proposition 2.1 implies that a subsequential limit of $\left(T_{j}, o_{j}\right)$ is recurrent a.s. By Rayleigh monotonicity, $G$ is recurrent a.s.

\section{Concluding Remarks}

\subsection{Limits of uniform spherical triangulations}

Let $M \in[6, \infty]$; and let $T_{j}^{M}$ be chosen randomly-uniformly among isomorphism classes of spherical triangulations with $j$ vertices and maximum degree at most $M$, and given $T_{j}^{M}$ 
let $o_{j}$ be chosen uniformly among the vertices of $T_{j}^{M}$.

Conjecture 3.1. The distributional limit of $\left(T_{j}^{M}, o_{j}\right)$ exists.

This holds when $M=6$, and then the limit is the hexagonal grid (since by Euler's formula there can be in this case at most 12 vertices with degree smaller than 6 ). After a first draft of the current paper has been distributed, a proof of the conjecture for the case $M=\infty$ (that is, no restriction on the degrees) has been obtained by Omer Angel and Oded Schramm. A paper with this result is forthcoming.

Assuming the conjecture for now, let $\left(T^{M}, o\right)$ denote the limit random rooted triangulation. Let $p_{n}\left(T^{M}\right)$ denote the probability that the simple random walk starting from $o$ will be at $o$ at time $n$, given $T^{M}$. Following the discussion in the introduction, it should be believed that $p_{n}\left(T^{M}\right)$ decays like $n^{-1}$ for almost all $T^{M}$. Theorem 1.1 shows that when $M<\infty$ the decay cannot be faster than $n^{\alpha}$ with $\alpha<-1$.

\subsection{Intrinsic Mass Transport Principle}

Proposition 2.2 implies that every distributional limit of unbiased random bounded degree triangulations of the sphere has at most two ends. (If $m \in \mathbb{N}$, the statement that a graph $G$ has $m$ ends is equivalent to the statement that $m$ is the maximum number of infinite components of $G \backslash W$ as $W$ ranges over finite subsets of $V(G)$. The definition of the space of ends is a bit more complicated, and can be found in many textbooks on pointset topology.) It is easy to verify that the proof applies also to the case where there is no uniform bound on the degrees, assuming that the limit graph is locally finite a.s. In fact, one can show that under the assumptions of Theorem 1.1, $G$ has at most two ends. Moreover, if $G$ is the distributional limit of unbiased finite rooted graphs, then a.s. if $G$ is recurrent it has at most two ends. This can be proved using an intrinsic version of the Mass Transport Principle (MTP). The extrinsic version of MTP [Häg97, BLPS99] in its simplest form says that $\sum_{x \in \Gamma} f(x, y)=\sum_{x \in \Gamma} f(y, x)$ where $\Gamma$ is a discrete countable group and $f: \Gamma \times \Gamma \rightarrow[0, \infty)$ is invariant under the diagonal action of $\Gamma$ on $\Gamma \times \Gamma$, $\gamma:(g, h) \rightarrow(\gamma g, \gamma h)$

We now briefly explain the intrinsic MTP. Suppose that $f(G, x, y)$ is a function which takes a graph $G$ and two vertices $x, y$ in $G$ and returns a non-negative number. We assume that $f$ is isomorphism invariant, in the sense that $f(\psi(G), \psi(x), \psi(y))=f(G, x, y)$, when $\psi$ is a graph isomorphism. Also assume that $f$ is measurable on the appropriate space of connected graphs with two distinguished vertices. Then $f$ will be called a transport function. (For example, if $g(G, v, u)$ is the degree of $v$ if $v$ neighbors with $u$ in $G$ and zero otherwise, then $g$ is a transport function.) A probability measure $\mu$ on $\mathcal{X}$ is said to satisfy the intrinsic MTP (IMTP) if for every transport function $f$ we have

$$
\mathbf{E}\left[\sum_{v} f(G, o, v)\right]=\mathbf{E}\left[\sum_{v} f(G, v, o)\right],
$$



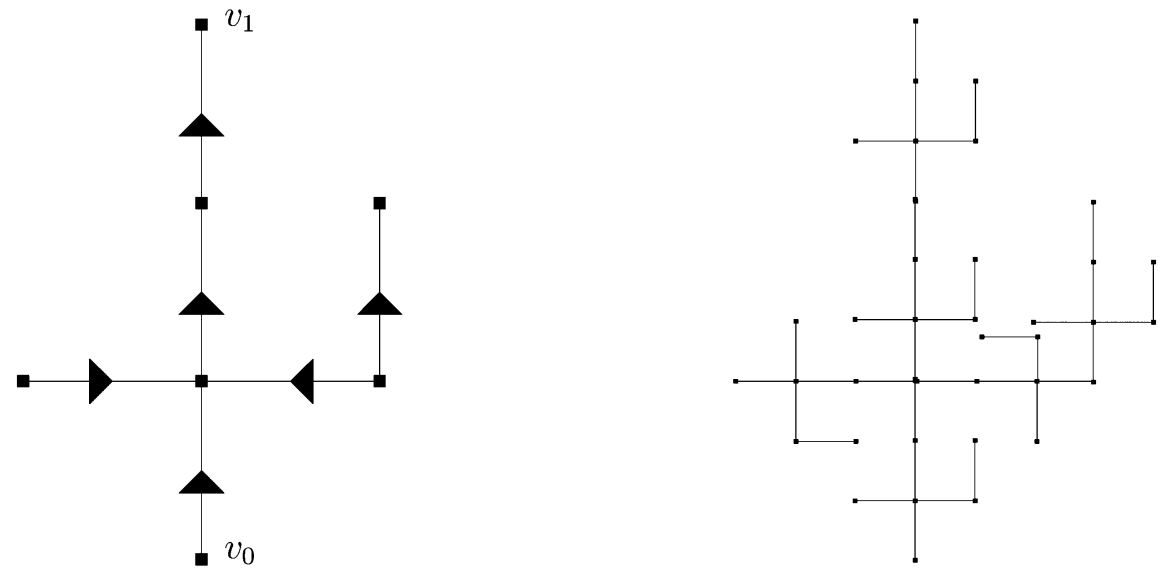

Figure 3.1: A subdivision rule for a tree.

where the sum on both sides is over all the vertices of $G$. It is easy to verify that every unbiased probability measure on finite graphs in $\mathcal{X}$ satisfies the IMTP. We claim that this also extends to weak limits of such measures. Indeed, let $\mu$ be a weak limit of Borel probability measures $\mu_{1}, \mu_{2}, \ldots$ on $\mathcal{X}$ satisfying the IMTP. Given a transport function $f$ and some $k>0$, let $f_{k}(G, v, u):=f(G, v, u)$ when $f \leq k$ and the distance from $v$ to $u$ in $G$ is at most $k$, and $f_{k}(G, v, u):=0$, otherwise. It is clear that both sides of (3.1) for $\mu$ and $f_{k}$ are the limits of the corresponding terms for $\mu_{j}$, hence they are equal. The fact that $\mu$ satisfies the IMTP now follows by taking the supremum with respect to $k$.

Many of the applications of MTP, such as appearing in [BLPS99], for example, can therefore be applied to (limits of finite) unbiased random graphs, we hope to further pursue this in a future work.

\subsection{Examples of triangulations with uniform growth}

It was observed by physicists [ADJ97] that for some random triangulations the average volume of balls of radius $r$ is $r^{4}$. This looks surprising and calls for an intuitive explanation. We can't provide one, but to shed some light on the geometry of random surfaces, we can construct for every $\alpha>1$ a triangulation of the plane for which every ball of radius $r$ has $r^{\alpha}$ vertices, up to a multiplicative constant.

The simplest such construction is based on a tree. Consider a finite tree $t_{1}$ with two distinct marked vertices $v_{0}, v_{1}$, both having degree 1 . Direct the edges of $t_{1}$ arbitrarily. Let $t_{2}$ be the tree obtained from $t_{1}$ by replacing each directed edge $\left[u_{0}, u_{1}\right]$ by a new copy of $t_{1}$, where $v_{0}$ replaces $u_{0}$ and $v_{1}$ replaces $u_{1}$. See Figure 3.1 for an example. Inductively, let $t_{n}$ be obtained from $t_{n-1}$ by replacing each edge of $t_{n-1}$ with a copy of $t_{1}$. Note that the maximum degree in $t_{n}$ is the maximum degree in $t_{1}$. Suppose that $t_{1}$ has $k$ edges and the distance from $v_{0}$ to $v_{1}$ in $t_{1}$ is $\Delta$. It is then clear that the diameter of $t_{n}$ is $\Delta^{n}$, 

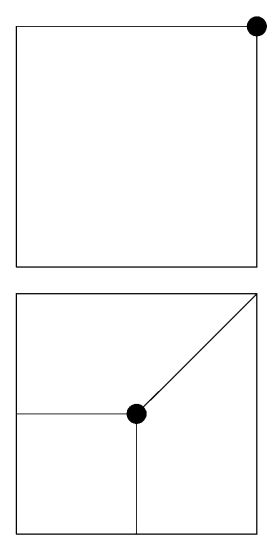

Figure 3.2: A subdivision rule and a resulting self-similar tiling.

up to an additive constant, and the number of edges of $t_{n}$ is exactly $k^{n}$. It follows that for $m \leq n$ every ball of radius $\Delta^{m}$ in $t_{n}$ has $k^{m}$ edges, up to a multiplicative constant, because $t_{n}$ is obtained by appropriately replacing each edge of $t_{n-m}$ by a copy of $t_{m}$. Then every ball of radius $r \leq \operatorname{diam}\left(t_{n}\right)$ in $t_{n}$ has about $r^{\log k / \log \Delta}$ vertices. We may pick a root in each $t_{n}$ and take a subsequential limit, to obtain a tree $t_{\infty}$ where every ball of radius $r$ has about $r^{\alpha}$ vertices, where $\alpha=\log k / \log \Delta$.

One can easily modify the construction to obtain a similar tree with growth $r^{\alpha}$ where $\alpha>1$ is not the ratio of logs of integers, by letting the replacement rule from $t_{n-1}$ to $t_{n}$ appropriately depend on $n$.

It is easy to make planar triangulations with similar properties. For example, suppose that the maximum degree in $t_{\infty}$ is $M$, and let $T$ be a triangulation of the sphere with at least $M$ disjoint triangles. Then we may replace each vertex $v$ of $t_{\infty}$ by a copy $T_{v}$ of $T$ and for each edge $[v, u]$ in $t_{\infty}$ glue a triangle in $T_{v}$ to a triangle in $T_{u}$, in such a way that every vertex of $T_{v}$ is glued to at most one other vertex. The resulting graph is the 1-skeleton of a planar triangulation, as required.

Another example which is somewhat similar but not tree-like appears in Figure 3.2. It is obtained by starting with a quadrilateral with a marked corner, subdividing it as in the figure to obtain three quadrilaterals with the interior vertex as the marked corner of each, and continuing inductively. The result is a map of the plane with quadrilateral faces and maximum degree 6 .

These examples answer Problem 1.1 from [Bab97]

Acknowledgment. We thank Bertrand Duplantier for introducing us to the fascinating subject of random spherical triangulations. 


\section{References}

$\left[\mathrm{AAJ}^{+}\right.$98] Jan Ambjørn, Konstantinos N. Anagnostopoulos, Lars Jensen, Takashi Ichihara, and Yoshiyuki Watabiki. Quantum geometry and diffusion. J. High Energy Phys., (11):Paper 22, 16 pp. (electronic), 1998.

[ADJ97] Jan Ambjørn, Bergfinnur Durhuus, and Thordur Jonsson. Quantum geometry. Cambridge University Press, Cambridge, 1997. A statistical field theory approach.

$\left[\mathrm{ANR}^{+}\right.$98] Jan Ambjørn, Jakob L. Nielsen, Juri Rolf, Dimitrij Boulatov, and Yoshiyuki Watabiki. The spectral dimension of 2D quantum gravity. J. High Energy Phys., (2):Paper 10, 8 pp. (electronic), 1998.

[Bab97] László Babai. The growth rate of vertex-transitive planar graphs. In Proceedings of the Eighth Annual ACM-SIAM Symposium on Discrete Algorithms (New Orleans, $L A, 1997)$, pages 564-573, New York, 1997. ACM.

[Ber92] Marcel Berger. Les placements de cercles. Pour la Science (French Scientific American), 176, 1992.

[BLPS99] I. Benjamini, R. Lyons, Y. Peres, and O. Schramm. Group-invariant percolation on graphs. Geom. Funct. Anal., 9(1):29-66, 1999.

[BS96] I. Benjamini and O. Schramm. Harmonic functions on planar and almost planar graphs and manifolds, via circle packings. Invent. Math., 126:565-587, 1996.

[dV91] Yves Colin de Verdière. Une principe variationnel pour les empilements de cercles. Inventiones Mathematicae, 104:655-669, 1991.

[GW00] Zhicheng Gao and Nicholas C. Wormald. The distribution of the maximum vertex degree in random planar maps. J. Combin. Theory Ser. A, 89(2):201-230, 2000.

[Häg97] Olle Häggström. Infinite clusters in dependent automorphism invariant percolation on trees. Ann. Probab., 25(3):1423-1436, 1997.

[HS95] Zheng-Xu He and O. Schramm. Hyperbolic and parabolic packings. Discrete Comput. Geom., 14(2):123-149, 1995.

[JS00] Johan Jonasson and Oded Schramm. On the cover time of planar graphs. Electron. Comm. Probab., 5:85-90, 2000. paper no. 10.

[Koe36] P. Koebe. Kontaktprobleme der Konformen Abbildung. Ber. Sächs. Akad. Wiss. Leipzig, Math.-Phys. Kl., 88:141-164, 1936.

[Mal99] V. A. Malyshev. Probability related to quantum gravitation: planar gravitation. Uspekhi Mat. Nauk, 54(4(328)):3-46, 1999.

[McC98] Gareth McCaughan. A recurrence/transience result for circle packings. Proc. Amer. Math. Soc., 126(12):3647-3656, 1998. 
[MP94] S. Malitz and A. Papakostas. On the angular resolution of planar graphs. SIAM J. Discrete Math., 7:172-183, 1994.

[MT90] Gary L. Miller and William Thurston. Separators in two and three dimensions. In Proceedings of the 22nd Annual ACM Symposium on Theory of Computing, pages 300-309. ACM, Baltimore, May 1990.

[RS87] Burt Rodin and Dennis Sullivan. The convergence of circle packings to the Riemann mapping. J. Differential Geom., 26(2):349-360, 1987.

[Sac94] Horst Sachs. Coin graphs, polyhedra, and conformal mapping. Discrete Math., 134:133-138, 1994.

[Sch99] Gilles Schaeffer. Random sampling of large planar maps and convex polyhedra. In Annual ACM Symposium on Theory of Computing (Atlanta, GA, 1999), pages 760-769 (electronic). ACM, New York, 1999.

[Ste99] Kenneth Stephenson. Approximation of conformal structures via circle packing. In N. Papamichael, St. Ruscheweyh, and E. B. Saff, editors, Computational Methods and Function Theory 1997, Proceedings of the Third CMFT Conference, volume 11, pages 551-582. World Scientific, 1999.

[Tho99] Robin Thomas. Recent excluded minor theorems for graphs. In Surveys in combinatorics, 1999 (Canterbury), pages 201-222. Cambridge Univ. Press, Cambridge, 1999.

[Tut62] W. T. Tutte. A census of planar triangulations. Canad. J. Math., 14:21-38, 1962. 